\title{
Diseño urbano para áreas devastadas
}

Además de sustentar una parte importante de las relaciones entre los babitantes de la ciudad (transporte, comunicación, intercambio), las obras de infraestructura también pueden resolver la relación entre la ciudad y el entorno donde se emplaza. Alejada cada vez más del plano de la dominación, esta relación debiera convertirse en una suerte de diálogo, articulado por caminos, diques, canales y drenajes. Palabras clave: Urbanismo, aluvión, inundaciones, Litoral Central,
Macuto, rehabilitación urbana,.

Much of the relationship among citizens is sustained by public works (in terms of transport, communication and exchange), and they can also resolve the relationship between the city and its location. Ever less a matter of domination, the relationship should become a kind of dialogue, structured by roads, ditches, canals and drains. Key words: Town planning, urban design, landslide, flooding.

Central Coast, Macuto, urban renewal.
Rehabilitación del Litoral Central de Venezuela ${ }^{1}$

Los asentamientos urbanos dependen del entorno natural donde se posan y crecen. Terremotos, deslaves, inundaciones, erupciones, avalanchas y tsunamis cambian el territorio y la vida de sus habitantes. Dependemos de la naturaleza, y el conocerla nos permitirá diseñar nuestras ciudades y pueblos para minimizar riesgos.

En 1999 el Litoral Central de Venezuela fue arrasado por aludes torrenciales que descendieron de la montaña que lo separa de Caracas; a solicitud del Estado, universidades nacionales trabajaron en el sector. Un equipo de la Universidad Metropolitana (UNIMET) junto a expertos de instituciones nacionales e internacionales, trabajaron para la rehabilitación de cinco áreas con alto valor inmobiliario, ambiental, recreacional y patrimonial: Macuto, Camurí Chico, Los Corales, Tanaguarena y Carmen de Uria. La meta era transformar esta tragedia en una oportunidad.

\section{Vulnerabilidad urbana}

Varios desastres naturales han modificado el área metropolitana de Caracas. Sólo en la historia reciente del Litoral están documentados deslaves en 1938, 1948, 1951, 1999 y 2005.

En 1999, lluvias torrenciales sobre las regiones norte-centro (Litoral Central) y norte-occidente

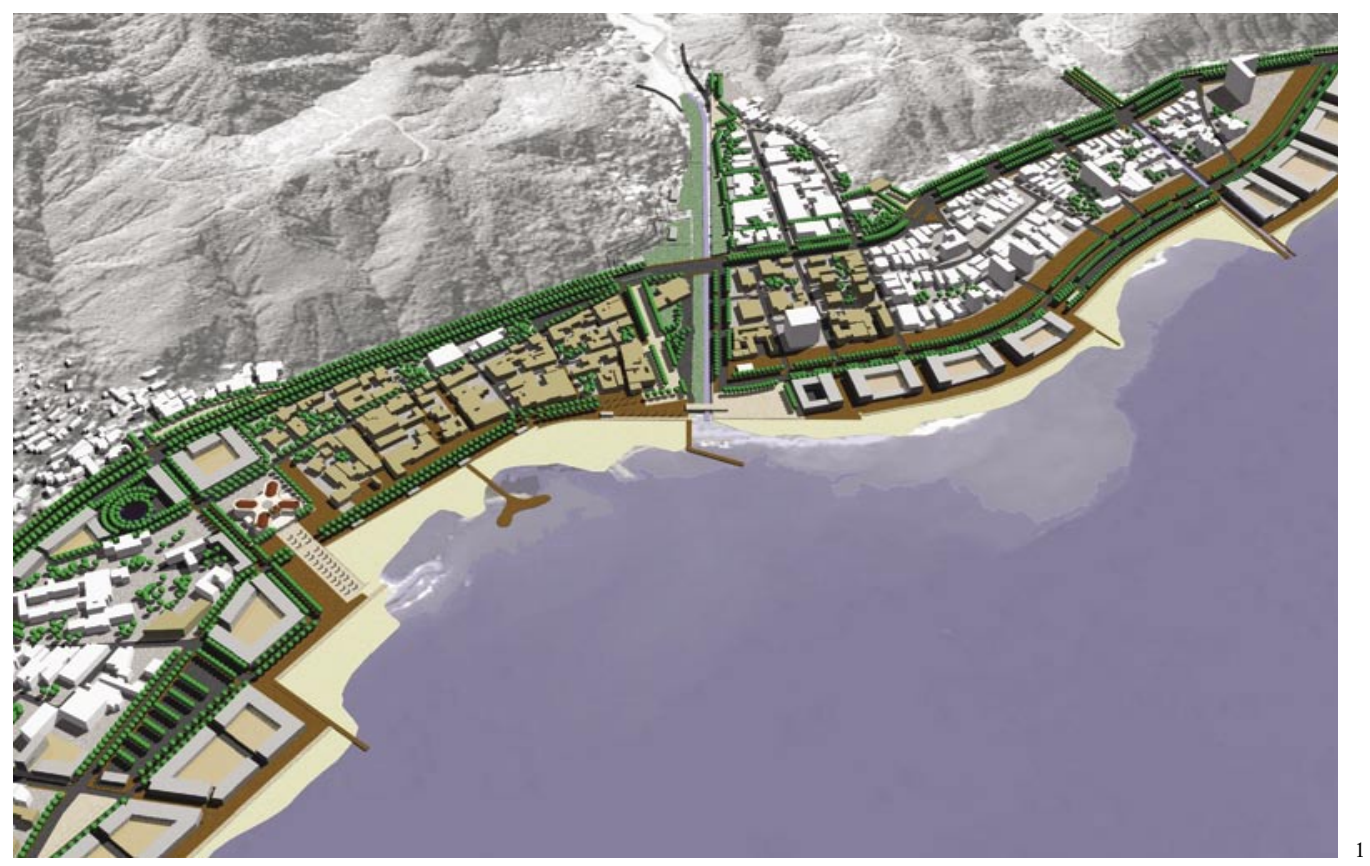

1 Vista aérea, propuesta para Macuto. Maestría en Diseño Urbano UNIMET

2 Propuesta para canales de recuperación ambiental y diques apaciguadores de la energía. Fuente: Maestría en Diseño Urbano UNIMET

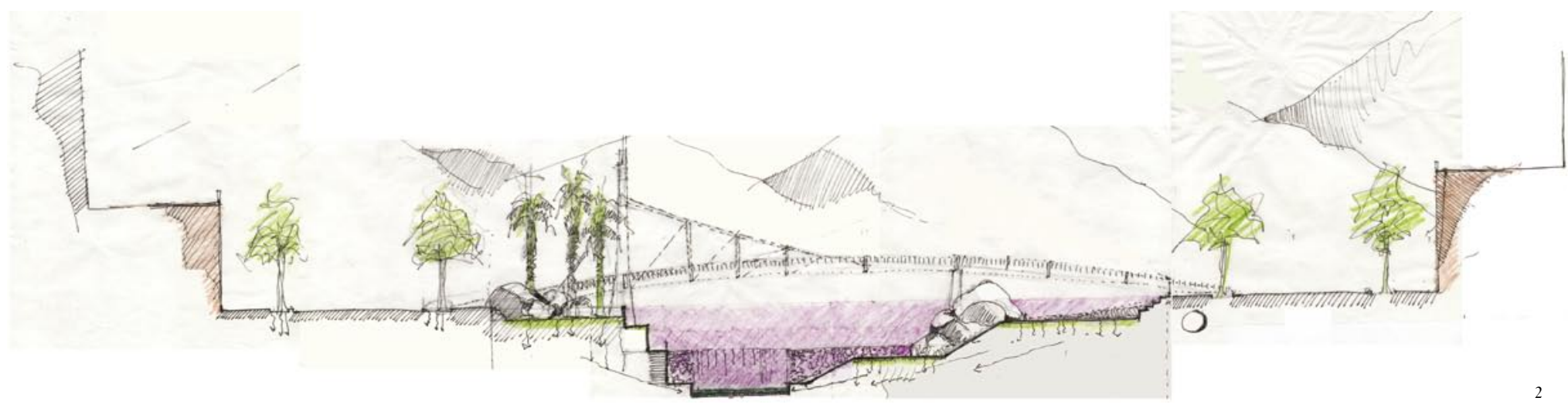


del país -durante veinte días aproximadamentegeneraron daños que el Consejo Nacional de la Vivienda estimó en 11.000 edificaciones residenciales afectadas y 80.000 personas desplazadas. Se calculan 15.000 fallecidos, aunque la cifra oficial no ha sido develada. La población residente estimada para entonces era de 350.000 habitantes más una población flotante de 1.000 .000 de habitantes durante los fines de semana.

En el 2005, otra vaguada generó 61 muertos, 22.000 damnificados, 70 desaparecidos y más de 38.000 viviendas afectadas, según el Ministerio de Interior y Justicia. Pese a los avances, el Litoral aún sigue vulnerable, esta vez por falta de la infraestructura óptima para minimizar riesgos aguas arriba: los trabajos propuestos por los expertos no fueron ejecutados según lo proyectado, y seis años después de la última emergencia es evidente la repercusión de los trazados de infraestructura (malos o inexistentes) en las zonas urbanas. Desechos, material pétreo, árboles y lodo acumulados alteraron de manera significativa $-y$ en algunos casos de forma permanente- la topografía de áreas pobladas próximas a las vertientes de los ríos.

La evacuación de población por la emergencia llevó a una ruptura del tejido social, trasladando a la población hacia Caracas y a otros asentamientos a lo largo y ancho del territorio. Esta política errada separó a las comunidades de su entorno, familiares, vecinos y lugares de trabajo.

\section{El Litoral Central de Venezuela}

Los núcleos urbanos del Litoral Central, que es una franja de aproximadamente $26 \mathrm{~km}$ de largo por $2 \mathrm{~km}$ de ancho, se sitúan en las planicies de inundación y los conos de deyección de los cursos de agua que descienden de la cordillera de cara al mar Caribe. Las tierras de menor pendiente y aptas de ser urbanizadas coinciden con las zonas de mayor riesgo desde el punto de vista hidrológico. Para ser ocupadas requieren la construcción de obras de ingeniería: escolleras, estabilización de suelos, canales, terrazas, viaductos, puentes y túneles.

En esta zona se encuentran el principal puerto y aeropuerto del país, centros históricos de gran valor patrimonial, áreas residenciales y servicios turísticos. Un tejido social de gran fuerza está asentado en esta franja urbana.

A pesar de estas fortalezas, los problemas se evidencian fácilmente: degradación ambiental, pobreza, congestionamiento vehicular durante días feriados, deficiencias en servicios básicos, precaria accesibilidad y ocupación ilegal del parque nacional El Ávila, con la consiguiente contaminación de quebradas y playas.

Aunque debía ser la puerta hacia el continente, estas condiciones impiden que el Litoral Central venezolano sea parte del mercado turístico del Caribe.

Era posible transformar una tragedia en una oportunidad

Las propuestas de la UNIMET para los sectores más afectados del Litoral Central -Macuto, Camurí, Los Corales, Tanaguarena y Carmen de Uria- buscaban solventar las deficiencias que antes de los aludes torrenciales presentaba el Litoral, al tiempo de crear nuevas directrices basadas en la minimización de la vulnerabilidad urbana.

Con la finalidad de exponer los criterios básicos de diseño urbano y trazados de infraestructura como instrumento para la minimización de riesgos, a continuación se exponen las propuestas para una de las zonas con mayor carga social e histórica: Macuto.

\section{Área patrimonial. Macuto - El Cojo}

Macuto, el primer balneario del país, aún conserva el trazado tradicional y su tejido sigue ocupado por posadas, residencias geriátricas, restaurantes y hoteles, entremezclados con casas

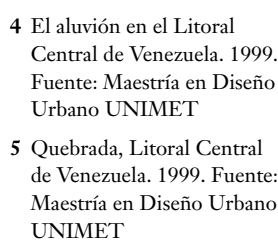

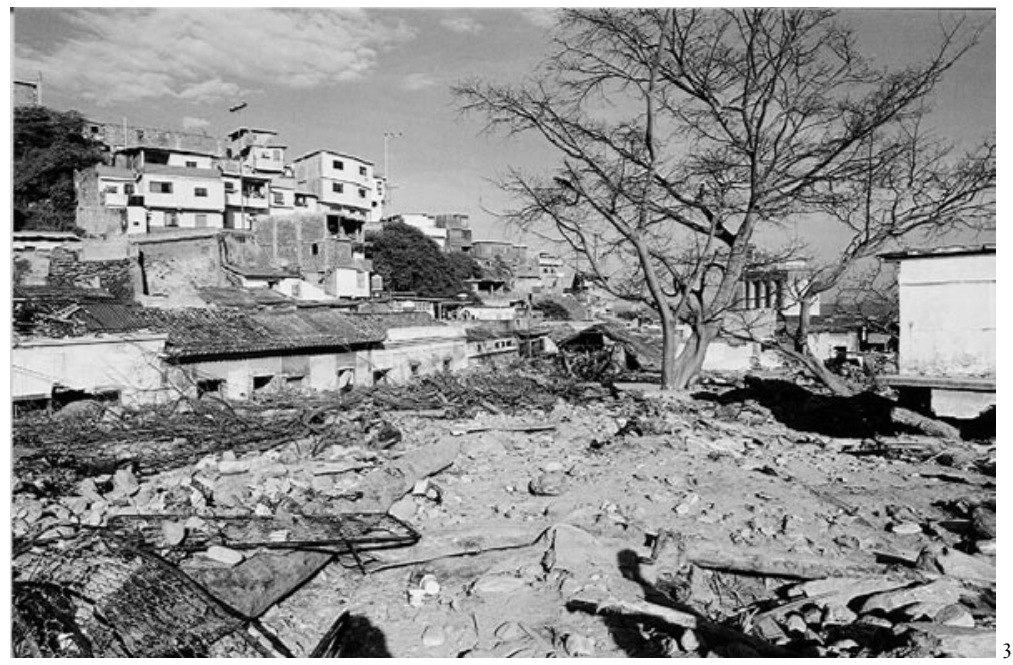

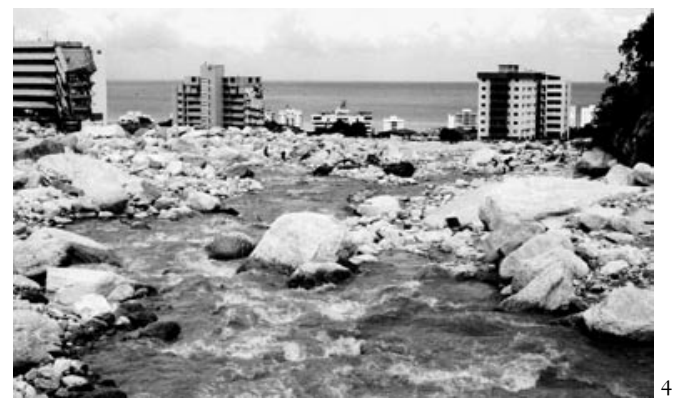


de familias de larga data en la zona y de nuevos residentes atraídos por ese ambiente pueblerino. En 1999 los ríos Macuto y el Cojo inundaron de barro, piedras, árboles y material orgánico el casco histórico. Desaparecieron playas y la costa avanzó en algunos puntos más de $250 \mathrm{~m}$ mar adentro.

"Ciudad balneario", la propuesta urbana para Macuto, buscaba minimizar riesgos y para esto se diseñaron:

Retenedores aguas arriba y canales aguas abajo. Asociados a oportunidades urbanas, turísticas y recreativas, e indispensables para reducir el aporte aluvional en las cuencas altas, se propuso la construcción de diques apaciguadores de la energía, que buscaban filtrar el material de arrastre descargando drenes a los canales aguas abajo -cuyo diseño debía estar integrado armoniosamente al nuevo escenario urbano-. La propuesta era complementada con programas de estabilización y reforestación.

Se plantearon trazados con curvaturas suaves para los canales aguas abajo, siguiendo el curso natural del agua. Para estos canales de recuperación ambiental, que consideraban su integración al ecosistema del lugar, se proponían una sección fija de concreto y una sección variable, con superficies que acentuaban el valor de este elemento de infraestructura como parte del sistema de espacios públicos recreacionales que vinculaban la costa con la montaña.

Es importante destacar que las quebradas y ríos solían ubicarse hacia los fondos de las parcelas, favoreciendo la ocupación gradual de los cauces y el depósito de basura o escombros. Por esta razón, se desconocía la verdadera ubicación de los cauces y el comportamiento de las aguas. Los nuevos conceptos para el diseño de los canales promoverían una cultura ciudadana en relación al agua y al disfrute del espacio abierto asociado a los cauces.

Nueva red vial en estrecha relación con las operaciones urbanísticas. El nuevo trazado vial resolvía la congestión vehicular, incluía sistemas eficientes de transporte público y facilitaba las nuevas operaciones urbanísticas. A tal efecto, su trazado aprovechaba las áreas ganadas al mar, así como aquellas zonas que no pueden ser reurbanizadas. Las secciones propuestas incluían: amplias aceras, tratamiento paisajístico en paseos peatonales, vías de servicio y/o canales preferenciales para el transporte público y áreas de estacionamiento. El trazado respondía a un sistema que enlazaba toda la franja de asentamientos del Litoral.

Nuevos desarrollos y planes de rehabilitación del patrimonio. Buscaban reafirmar la vocación recreacional y residencial de estas áreas, rehabilitar y reordenar el frente costero $y$ sus edificaciones patrimoniales, extender el paseo peatonal hacia el oeste del casco central, reacondicionar playas y crear nuevas.

Viviendas de sustitución. Se propusieron nuevas viviendas para reubicación de residentes de áreas inestables o de espacios requeridos para la construcción de nueva infraestructura. Estas edificaciones se planteaban como viviendas productivas para integrar de manera funcional $y$ espacial los diferentes asentamientos.

Calles como limites de crecimiento urbano. Nuevas vías en el borde del parque nacional El Ávila controlarían las expansiones sobre esta zona, acompañado de equipamientos vecinales (preescolares, recreativos y otros).

\section{Infraestructura y ciudad}

La puesta en práctica de los proyectos urbanos no sólo depende de un buen plan. La interacción y el trabajo coordinado entre el sector público, el privado y la comunidad son claves importantes para hacer factibles las propuestas.

Días después de la tragedia de 1999, el Litoral Central demostró tener un fuerte tejido social. La comunidad volvió a su entorno a remover escombros, despejar calles y ayudar a sus vecinos en el marco del dolor de la pérdida de familiares, amigos y bienes. Meses después y en conjunto con el Estado, instituciones internacionales, universidades y expertos, presentaron los criterios y propuestas para la rehabilitación del área.

Cuatro años después de estos eventos, se habían adelantado labores de construcción de diques y canales aguas arriba, con graves errores de construcción. Los primeros canales construidos aguas abajo no cumplen con los criterios ecológicos propuestos, perdiendo la oportunidad para el desarrollo de espacios públicos y el enlace entre el mar y la montaña.

En febrero de 2005 se generaron vaguadas sobre Venezuela y otros países, que ocasionaron inundaciones en Los Andes, Caracas y la costa, repitiendo la tragedia en el Litoral.

Una parte considerable de la población sigue viviendo fuera del Litoral. El Estado ha reubicado estos desplazados fuera de su hábitat natural, creando ghettos alejados de sus fuentes de empleo y su entorno social. Zonas de alto riesgo están siendo ocupadas por la población damnificada con bajo poder adquisitivo, aumentando la densidad de los asentamientos espontáneos. En parte esto se debe a la falta de respuestas del Estado en cuanto a planes de vivienda social en conjuntos urbanos de calidad en la zona.
Los proyectos urbanos para la ciudad contemporánea deben entender su geografía y detectar los espacios de oportunidad muchas veces ignorados: bordes de ríos, frentes costeros y tierras ganadas al mar. Estos espacios permitirían diversificar funciones, densificar, reciclar y mejorar la calidad de vida de habitantes y visitantes. ARQ
Bibliografía: Cilento, Alfredo; "Sobre la vulnerabilidad urbana de Caracas". Disponible en www.revele.com.ve/ pdf/revista venezolana de economia_y ciencias sociales/ volsn3/pag13.pdf / Universidad Metropolitana; Rebabilitación de el Litoral Central. Oscar Todtmann editores, Caracas, 2001. / VV.AA.; Fuerte vaguada 2005 en Venezuela. En http://www.lmmeteoven.org/vaguada feb05.html. 